\title{
Sleep Health and Serious Psychological Distress: A Nationally Representative Study of the United States among White, Black, and Hispanic/Latinx Adults
}

This article was published in the following Dove Press journal:

Nature and Science of Sleep

Samuel J Goldstein (iD)

Symielle A Gaston $\mathbb{D}^{2}$

John A McGrath $\mathbb{D}^{3}$

Chandra $L$ Jackson $\mathbb{I D}^{2,4}$

'The University of North Carolina at Chapel Hill, Department of

Environmental Sciences and Engineering, Chapel Hill, NC, USA; ${ }^{2}$ Department of Health and Human Services,

Epidemiology Branch, National Institute of Environmental Health Sciences, National Institutes of Health, Research Triangle Park, NC, USA; ${ }^{3}$ Social \& Scientific Systems, Inc, Durham, NC, USA; ${ }^{4}$ Division of Intramural Research, National Institute on Minority Health and Health Disparities, National Institutes of Health, Department of Health and Human Services, Bethesda, MD, USA
Correspondence: Chandra L Jackson III T.W. Alexander Drive, MD A3-05, Research Triangle Park, NC 27709

Tel +984-287-370।

Fax $+30 \mathrm{I}-480-3290$

Email Chandra.Jackson@nih.gov
Purpose: Prior studies investigating the relationship between sleep and serious psychological distress (SPD) have lacked racial/ethnic diversity and generalizability. We investigated associations between sleep and SPD among a large, nationally representative, and racially/ ethnically diverse sample of US adults.

Methods: We pooled cross-sectional data from the 2004 to 2017 National Health Interview Survey. Participants self-reported sleep duration and sleep disturbances (eg, trouble falling and staying asleep). SPD was defined as a Kessler Psychological Distress Scale (K6) score $\geq 13$. Adjusting for sociodemographic, health behavior, and clinical characteristics, we used Poisson regression with robust variance to estimate prevalence ratios (PRs) and 95\% confidence intervals (CIs) of SPD for each sleep characteristic, overall and by race/ethnicity.

Results: Among 316,840 participants, the mean age \pm standard error was $46.9 \pm 0.1$ years, $52 \%$ were women, $75 \%$ were non-Hispanic (NH)-White, $16 \%$ NH-Black, and $9 \%$ Hispanic/ Latinx. The prevalence of SPD was 3.4\% for NH-Whites, $4.1 \%$ for NH-Blacks, and $4.5 \%$ for Hispanics/Latinxs. Participants with $<7$ hours versus 7-9 hours of sleep duration were more likely to have SPD, and the magnitude of the association was strongest among NH-Black participants $\left(\mathrm{PR}_{\mathrm{NH}-\mathrm{Blacks}}=3.50\right.$ [95\% CI: 2.97-4.13], PR Hispanics/Latinx $=2.95$ [2.42-3.61], and $\mathrm{PR}_{\mathrm{NH}-\mathrm{Whites}}=2.66$ [2.44-2.89]). Positive associations between sleep disturbances and SPD were generally stronger among NH-Black and Hispanic/Latinx compared to NH-White adults.

Conclusion: Poor sleep health was positively associated with SPD, and the magnitude of the association was generally stronger among racial/ethnic minorities. Future investigations should prospectively focus on the determinants and health consequences of SPD attributable to objectively measured sleep across racial/ethnic groups.

Keywords: sleep initiation and maintenance disorders, sleep, psychological stress, health status disparities, continental population groups, race factors

\section{Introduction}

The 2013 Global Burden of Disease Report identified five types of mental illness (ie, major depression, anxiety disorders, schizophrenia, dysthymia, and bipolar disorder) among the leading contributors of years lived with disability globally. ${ }^{1}$ Experts also estimate that hundreds of billions of dollars are lost each year due to the detrimental effects of mental illness. ${ }^{2,3}$ Depression and anxiety, which are often referred to collectively as serious psychological distress (SPD), contribute the most to the burden 
caused by mental illness and are, therefore, of particular importance. Individuals with SPD and related disorders have impaired physical and mental functioning, more work absenteeism, and greater use of health-care services. ${ }^{4}$

Sleep is a complex physiological and behavioral process that contributes importantly to physical and mental health. In 2014, the Center for Disease Control and Prevention estimated that roughly one-third of all US adults get less than the recommended 7 hours of sleep in a 24-hour period. ${ }^{5}$ Insufficient sleep may contribute to SPD through dysregulation of the hypothalamic-pituitaryadrenal (HPA) axis and over activation of the sympathetic nervous system. Such biological mechanisms are supported by studies demonstrating that 24-hour urinary free cortisol, norepinephrine, and catecholamine metabolite levels were higher in patients with insomnia and objective sleep disturbances as compared to controls. ${ }^{6-9}$

Further, epidemiologic evidence also supports that insufficient sleep may increase risk of SPD across a variety of study populations (eg, the elderly, adolescents, and couples). ${ }^{10-13}$ However, most prior studies of the association between sleep and SPD had relatively small sample sizes and were conducted among special populations (eg, battered women, medical students, military personnel, and natural disaster survivors) exposed to particularly stressful environments. $^{14-17}$ Consequently, the findings from these studies are likely nongeneralizable to the general population. Of the few prior nationally representative studies of the US, only sleep duration was assessed, which is not comprehensive. ${ }^{18-21}$ Despite this fact, these studies have shown that the association between insufficient sleep and SPD tend to be stronger among racial/ethnic minority groups.

To address these gaps in the literature, we sought to determine associations between sleep duration as well as sleep disturbances and SPD among a large sample of racially/ethnically diverse adults in the US. We hypothesized that poor sleep would be positively associated with SPD and that non-Hispanic Black as well as Hispanic/ Latinx (a gender-neutral neologism) participants would have both higher levels of poor sleep health and SPD along with a stronger association between poor sleep and SPD compared to their non-Hispanic White counterparts.

\section{Methods}

\section{National Health Interview Survey}

Data for this analysis were collected from the 2004 to 2017 National Health Interview Survey (NHIS). The
NHIS is a series of annual cross-sectional, household surveys conducted via computer-assisted, in-person interviews among non-institutionalized US civilians. NHIS uses a three-stage stratified cluster probability sampling design to obtain a nationally representative sample. All publicly/freely available NHIS data has been extensively reviewed by the National Center for Health Statistics' Disclosure Review Board to protect the confidentiality of survey participants. The final response rate (2004-2017) for sampled adults was 61.9\% (range: 53.0-72.5\%). Each study participant provided informed consent. A further detailed description of NHIS procedures has been published elsewhere. ${ }^{22}$ Our study was considered exempt by the institutional review board (IRB) at the National Institute of Environmental Health Sciences because deidentified, publicly available data are not classified as human subjects research requiring IRB approval.

\section{Study Population}

Adults who self-identified as a race/ethnicity other than non-Hispanic (NH)-White, NH-Black, and Hispanic/ Latinx $(n=79,367)$ were excluded from our analysis due to small sample sizes. Because research has demonstrated that sleep and lifetime risk of mental disorder differs by nativity and acculturation, participants who specified that they were born outside of the US $(n=160,183)$ were excluded as beyond the scope of this study. ${ }^{23-25}$ Participants were also excluded if they had missing data on sleep duration $(n=7,819)$, sleep disturbance $(n=1,064)$ (measured from 2013 to 2017), or Kessler Psychological Distress Scale (K6) questions $(n=2,809)$. Our final analytic sample comprised 316,840 adults $(\geq 18$ years old: Supplemental Figure 1).

\section{Exposure Assessment: Sleep Characteristics Sleep Duration}

Sleep duration was assessed in all participants between survey years 2004 and 2017. Participants' estimates of overall time spent asleep were assessed with the question "On average, how many hours of sleep do you get in a 24hour period?". Interviewers were instructed to report hours of sleep in whole numbers, rounding values of $\geq 30$ minutes up to the nearest hour and rounding values $<30$ minutes down to the nearest hour. Sleep duration categories were: short ( $<7$ hours), recommended (7-9 hours), and long ( $>9$ hours). ${ }^{26}$ 


\section{Sleep Disturbances}

NHIS data on sleep disturbances were available for years 2013-2017. Sleep disturbances were assessed by asking participants (separately) how often in the past week they experienced trouble falling asleep, trouble staying asleep, felt rested upon awakening, and took sleep medication. If participants indicated that they experienced trouble falling asleep or trouble staying asleep $\geq 3$ days per week, they were categorized as having the respective sleep disturbance. Participants indicating that they did not wake feeling rested $\geq 3$ days per week were categorized as waking feeling unrested. Sleep medication use was based on selfreported use $\geq 3$ days per week.

\section{Outcome Assessment: Serious Psychological Distress}

SPD was assessed using the Kessler Psychological Distress Scale (K6). The K6 is a validated and frequently used screening tool for serious mental illness that has high specificity across racial/ethnic groups. ${ }^{27}$ Participants were asked how often they experienced symptoms of depression (ie, sadness, hopelessness, effort, or worthlessness) or anxiety (ie, nervous or restless/fidgety) in the past 30 days. Responses for each symptom ranged from 0 ("None of the time") to 4 ("All of the time"). Total scores ranged from 0 to 24 with higher scores representing higher levels of SPD. Consistent with the literature, participants with a total score $\geq 13$ were categorized as having SPD. ${ }^{27}$

\section{Potential Confounders}

Potential sociodemographic confounders included age (18-49, 50-64, and 65+ years), sex/gender (men/ women), educational attainment ( $<$ high school, high school, some college, and $\geq$ college graduate), annual household income $(<\$ 35,000, \$ 35,000$ to $<\$ 75,000$, and $\geq \$ 75,000$ ), living in poverty (reported total family income $<100 \%$ Federal Poverty Level or $\geq 100 \%$ Federal Poverty Level), occupational class (professional/management, support services, or laborers), employment status (yes/no), married/living with a partner (yes/no), and region of residence (South, Midwest, Northeast, or West). We considered health behaviors as potential confounders: smoking status (never, former, current), heavy alcohol consumption ( $\geq 2$ drinks/day for women; $\geq 3$ drinks/day for men), and physical activity (none, low, high). ${ }^{28}$ Participants who engaged in at least some level of activity and who provided a specific number of activity bouts were dichotomized at the midpoint of these bouts and classified as having activity levels of "low" or "high." Participants reporting "never" or "unable to do this type activity" were categorized as "none." Regarding clinical characteristics, self-reported measures of height and weight were used to estimate body mass index (BMI). BMI was categorized as underweight $\left(<18.5 \mathrm{~kg} / \mathrm{m}^{2}\right)$, normal weight (18.5$\left.<25.0 \mathrm{~kg} / \mathrm{m}^{2}\right)$, overweight $\left(25.0-<30.0 \mathrm{~kg} / \mathrm{m}^{2}\right)$, and obese $\left(\geq 30.0 \mathrm{~kg} / \mathrm{m}^{2}\right) .{ }^{29}$ Participants were separately asked if they had ever been told by a doctor or other health professional that they had hypertension (also called high blood pressure), diabetes/prediabetes, dyslipidemia, heart disease or cancer. ${ }^{28}$ Rather than considering each health behavior and clinical characteristic separately, a dichotomized measure of "ideal" cardiovascular health (yes vs no) was estimated using the following criteria: never smoked or quit smoking $>12$ months prior to interview; high levels of physical activity; normal BMI; and report of no prior diagnosis of dyslipidemia, hypertension, or diabetes/prediabetes. ${ }^{30}$ Additionally, we considered self-rated health ("excellent/ very good", "good", or "fair/poor") as part of a sensitivity analysis.

\section{Potential Moderators: Race/Ethnicity}

Participants were asked, "What race or races do you consider yourself to be?" They then self-identified as one or more of the following categories: White, Black/African American, American Indian/Alaskan native, Asian, or multiple races. Further, participants were asked, "Do you consider yourself to be Hispanic or Latino?" Based on these responses, participants who self-identified as White or Black and did not consider themselves to be Hispanic or Latino were categorized as NH-White and NH-Black (hereafter White and Black) while all others were considered Hispanic/Latinx. Whites comprised the largest sample size and, therefore, were used as the comparison group to confer greater statistical stability.

\section{Statistical Analysis}

Fourteen years of NHIS data (2004-2017) were merged by the Integrated Health Interview Series. ${ }^{31}$ Continuous variables were expressed as means \pm standard errors (SE), and categorical variables were expressed as weighted percentages derived after applying direct standardization to the sample using the age structure of the 2010 Census population where age groups were categorized as $18-49,50-64$, and $65+$ years. All analyses included sampling weights, which represent the inverse probability of selection into 
the sample, to account for unequal probability of selection resulting from the sampling design, nonresponse, and oversampling of the elderly and racial/ethnic minorities. We computed average sampling weights by dividing annual sampling weights by the number of survey years pooled. Thus, for our analysis of sleep disturbances that were available for years 2013-2017, we used sampling weights adjusted to a 5-year period whereas for all other analyses we used sampling weights adjusted to a 14-year period. We estimated descriptive statistics overall, by race/ ethnicity, and by SPD status. We then used Poisson regression with robust variance to estimate prevalence ratios (PRs) and 95\% confidence intervals (CIs) of SPD for each sleep characteristic, overall and by race/ethnicity. ${ }^{32}$ We also used Poisson regression models with robust variance estimation to compare racial/ethnic minorities who reported short, recommended, and long sleep to Whites who reported the recommended amount of sleep. All models were adjusted for the following potential confounders: age, sex/gender, educational attainment, annual household income, occupational class, employment status, alcohol consumption, and "ideal" cardiovascular health. ${ }^{19-21,33}$ A two-sided p-value of 0.05 was used to determine statistical significance.

As part of a sensitivity analysis to examine racial/ ethnic variation in the association between poor sleep health and SPD, we stratified Poisson regression models with robust variance by self-rated health (excellent/very good/good vs fair/poor). Poor health or disease (which are more prevalent in the elderly) may influence the relationship between sleep health and SPD within and across racial/ethnic groups. ${ }^{34} \mathrm{R}$, version 3.6.2, software ( $\mathrm{R}$ Foundation for Statistical Computing, Vienna, Austria) was used for all analyses, and a two-sided p-value of 0.05 was used to determine statistical significance.

\section{Results}

\section{Study Population Characteristics Overall,} by Race/Ethnicity, and by SPD

Among 316,840 participants, 3.6\% met the criteria for SPD. The mean age \pm standard error was $46.9 \pm 0.08$ years, 52\% were women, 75\% were White, 16\% were Black, $9 \%$ were Hispanic/Latinx, $9 \%$ had $<$ high school education, $32 \%$ had an annual household income $<\$ 35,000$, and $11 \%$ had "ideal" cardiovascular health (Table 1). Compared to participants without SPD, Whites with SPD were more likely to identify as women. The prevalence of unfavorable characteristics (eg, low socioeconomic status, harmful health behaviors, adverse clinical conditions) among Whites was generally lower than the prevalence of unfavorable characteristics among Black and Hispanic/Latinx participants. For instance, Blacks (17\%) and Hispanics/Latinxs (19\%) were more likely than Whites to attain a less than high school education (8\%). Both in the overall population and across racial/ethnic groups, there was a higher prevalence of unfavorable characteristics among participants with SPD compared to the participants without SPD. For instance, the prevalence of current smoking was higher among participants with vs without SPD in the overall (40\% vs $18 \%$ ), White (41\% vs $19 \%$ ), Black (39\% vs 19\%), and Hispanic/Latinx (32\% vs $14 \%)$ populations.

\section{Prevalence of Sleep Characteristics by Race/Ethnicity and SPD}

As illustrated in Figure 1, the prevalence of short sleep, long sleep, and sleep disturbances was greater among participants with vs without SPD in the overall population and for each race/ethnicity. Furthermore, Black participants had the largest absolute difference in the prevalence of short sleep duration among those with vs without SPD (61\% vs $36 \%$ compared to $50 \%$ vs $28 \%$ among Whites and $51 \%$ vs $31 \%$ among Hispanics/Latinxs).

\section{Associations Between Sleep Duration and SPD}

As shown in Figure 2 and Supplemental Table 1, nonrecommended sleep duration was positively associated with SPD overall and for each race/ethnicity. The association between short sleep duration ( $<7$ hours) and SPD was strongest among Black participants: Black participants who reported short sleep had over a three-fold higher prevalence of SPD compared to Black participants who reported recommended sleep duration $(\mathrm{PR}=3.50$ [95\% CI: 2.97-4.13] vs $\mathrm{PR}=2.66$ [95\% CI: 2.44-2.89] among Whites and $\mathrm{PR}=2.95$ [95\% CI: 2.423.61] among Hispanics/Latinxs). Although the confidence interval overlapped with those of other races/ethnicities, the magnitude of the association between long sleep duration ( $>9$ hours) and SPD was strongest among White participants $(\mathrm{PR}=3.36$ [95\% CI: 2.96-3.81] vs $\mathrm{PR}=2.27 \quad[95 \%$ CI: 1.70-3.03] among Blacks and $\mathrm{PR}=2.71 \quad[95 \%$ CI: 1.82-4.02] among Hispanics/ Latinxs). 


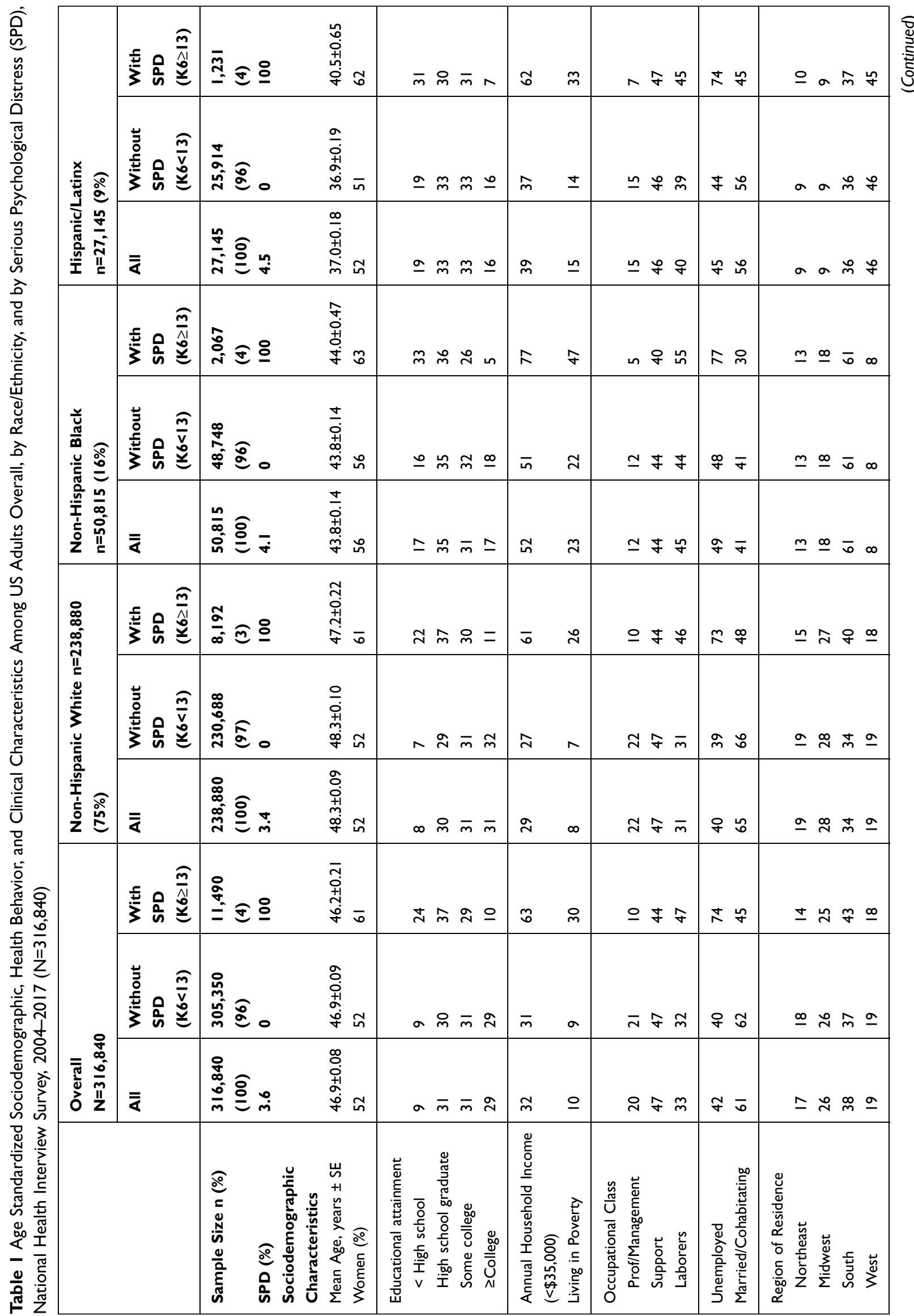




\begin{tabular}{|c|c|c|c|c|c|c|c|c|c|c|c|}
\hline \multirow{3}{*}{ 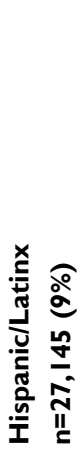 } & 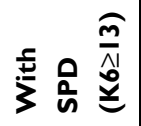 & & n & 홍 & พ $\stackrel{\sim}{\sim} \tilde{m}$ & $\infty$ & $\stackrel{\infty}{n} \simeq \dot{\sim}$ & & 유 & $\stackrel{ \pm}{m}$ & $\mathscr{T}$ \\
\hline & 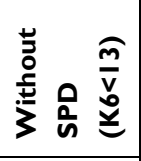 & & $\bar{m}$ ถ & $\bar{N} \dot{\sim} \stackrel{\sim}{m} \wedge$ & $\tilde{0} \boldsymbol{\sim} \pm$ & in & 우 $\stackrel{m}{q}$ & & $\stackrel{\sim}{\sim}$ & $\stackrel{\infty}{m}$ & m \\
\hline & $\bar{\varangle}$ & & $\bar{m} \quad t$ in & $\tilde{\sim} \sim \tilde{m} a$ & 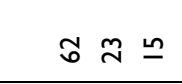 & in & 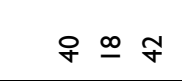 & & $\stackrel{i}{i}$ & $\stackrel{\infty}{m}$ & $\stackrel{m}{n}$ \\
\hline \multirow{3}{*}{ 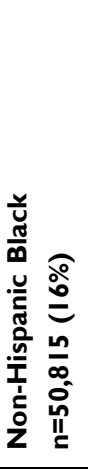 } & 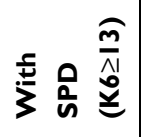 & & $\overline{0}$ 응 & oำ & 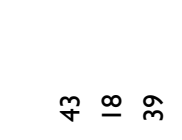 & $=$ & $\tilde{0} \simeq \approx$ & & $\stackrel{\sim}{\sim}$ & $\stackrel{\infty}{\sim}$ & $\stackrel{\circ}{f}$ \\
\hline & 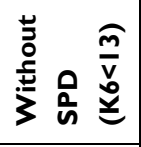 & & $\stackrel{\infty}{\infty} \stackrel{\infty}{\infty}$ & 유 㫕 & $\bar{\sigma} a \underline{a}$ & n & $\hat{f}=\stackrel{m}{0}$ & & స & $\dot{m}$ & q \\
\hline & $\overline{\bar{\alpha}}$ & & $\hat{m}$ in 0 & $\approx \approx \stackrel{n}{m} \infty$ & $\overline{0}$ 요 & 0 & 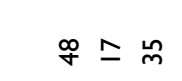 & & $\stackrel{2}{\sim}$ & $\dot{m}$ & $\bar{\sigma}$ \\
\hline \multirow{3}{*}{ 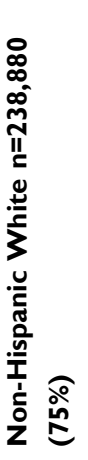 } & 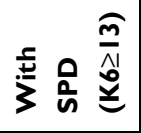 & & ㅇํ $\hat{m} \underline{m}$ & น & m & 으 & 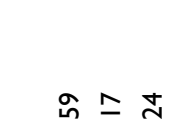 & & $\bar{m}$ & సે & $\bar{\sigma}$ \\
\hline & 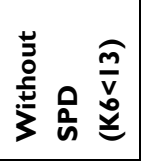 & & 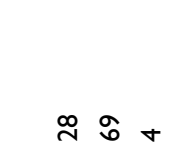 & 읏 $\stackrel{n}{n}$ 으 & 亡๋ & $a$ & $m \propto$ & & $\stackrel{\infty}{m}$ & $\stackrel{m}{m}$ & $\stackrel{\infty}{\sim}$ \\
\hline & $\overline{\bar{\alpha}}$ & & $\stackrel{\sim}{\infty} \stackrel{\infty}{\sigma}+$ & $\bar{N} \stackrel{m}{\circ}=$ & กิ & $a$ & 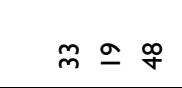 & & $\stackrel{\circ}{m}$ & $\stackrel{\circ}{m}$ & $\stackrel{\infty}{\sim}$ \\
\hline \multirow{4}{*}{ 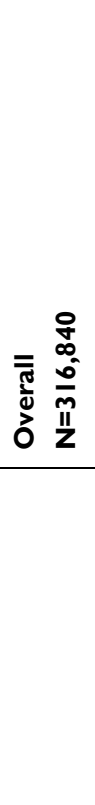 } & 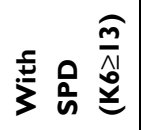 & & 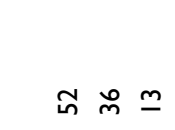 & ㅇ হ & 능 눙 웅 & 으 & 으 & & నે & సิ & $\mathcal{Z}$ \\
\hline & 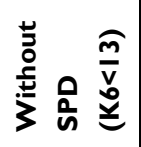 & & సิ ธิ & $\underline{a} \underset{\sim}{\infty} \stackrel{n}{m}$ 으 & นก น & $\infty$ & $\stackrel{m}{m} \underline{a}$ & & $\stackrel{m}{m}$ & ల & 이 \\
\hline & $\overline{\bar{\alpha}}$ & & ర : & $\bar{N}$ సి & นำ & $\infty$ & 의 & & $\dot{m}$ & $\stackrel{m}{m}$ & 이 \\
\hline & & 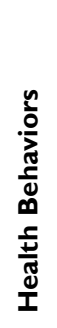 & 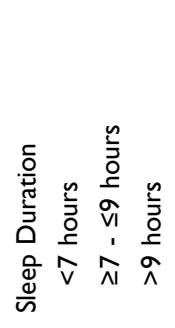 & 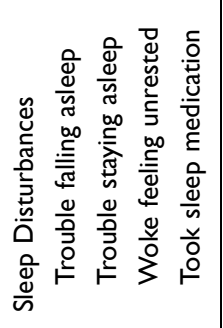 & 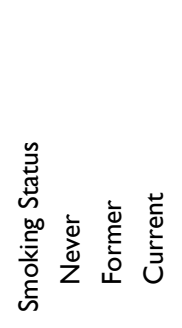 & 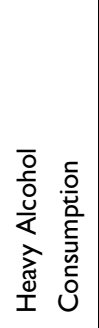 & 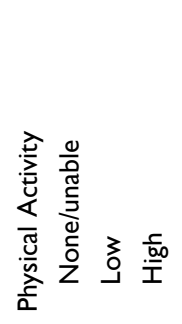 & 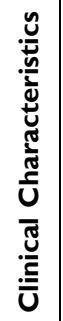 & 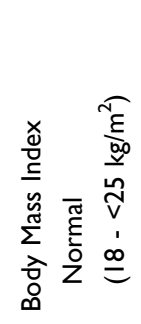 & 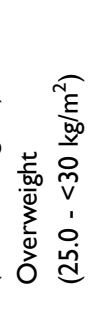 & 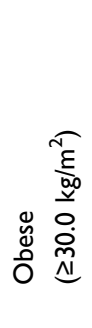 \\
\hline
\end{tabular}




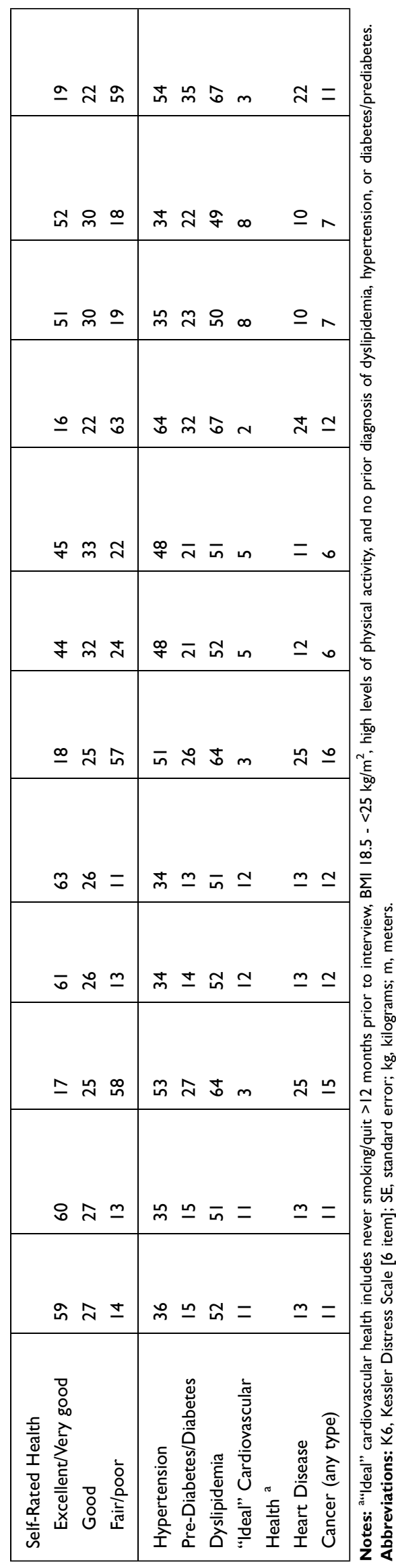

\section{Associations Between Sleep Disturbances and SPD}

Also shown in Figure 2 and Supplemental Table 1, sleep disturbances were positively associated with SPD overall and for each race/ethnicity. Excluding the association between woke not feeling rested and SPD for the Hispanic/Latinx population, prevalence ratios for the relationship between sleep disturbances and SPD among racial/ethnic minority groups were larger than those observed among Whites. For instance, the positive association between trouble falling asleep and SPD was stronger among Hispanic/Latinx participants $(\mathrm{PR}=9.10[95 \%$ CI:6.46-12.81]) compared to the positive association among Whites ( $\mathrm{PR}=4.88$ [95\% CI:4.29-5.55]). The positive association between trouble staying asleep and SPD was stronger among Black participants $(\mathrm{PR}=6.50[95 \%$ CI:4.97-8.51]) compared to the positive association among Whites ( $\mathrm{PR}=4.21$ [95\% CI:3.72-4.75]).

\section{Racial/Ethnic Comparison of SPD Prevalence Within Short, Recommended, and Long Sleep Duration Categories}

As shown in Figure 3 and Supplemental Table 2, compared to Whites who reported recommended sleep duration, Blacks and Hispanics/Latinx who reported either short or long sleep were more likely to have SPD. Compared to Whites who reported recommended sleep duration, Blacks who also reported recommended sleep were $38 \%$ less likely to have SPD ( $\mathrm{PR}=0.62[95 \%$ CI:0.53-0.73]). Hispanic/Latinxs who reported recommended sleep were also less likely to have SPD compared to Whites who reported recommended sleep ( $\mathrm{PR}=0.84$ [95\% CI:0.70-1.01]).

\section{Sensitivity Analysis}

Upon investigating the sleep-SPD association stratified by excellent/very good/good vs fair/poor self-rated health, we found that all associations between sleep health and SPD were stronger among those with excellent/very good/good compared to fair/poor self-rated health (Supplemental Table 3).

\section{Discussion}

In this large nationally representative study of White, Black, and Hispanic/Latinx adults in the US, we found that participants who reported $<7$ hours versus $7-9$ hours of sleep duration were more likely to be classified 


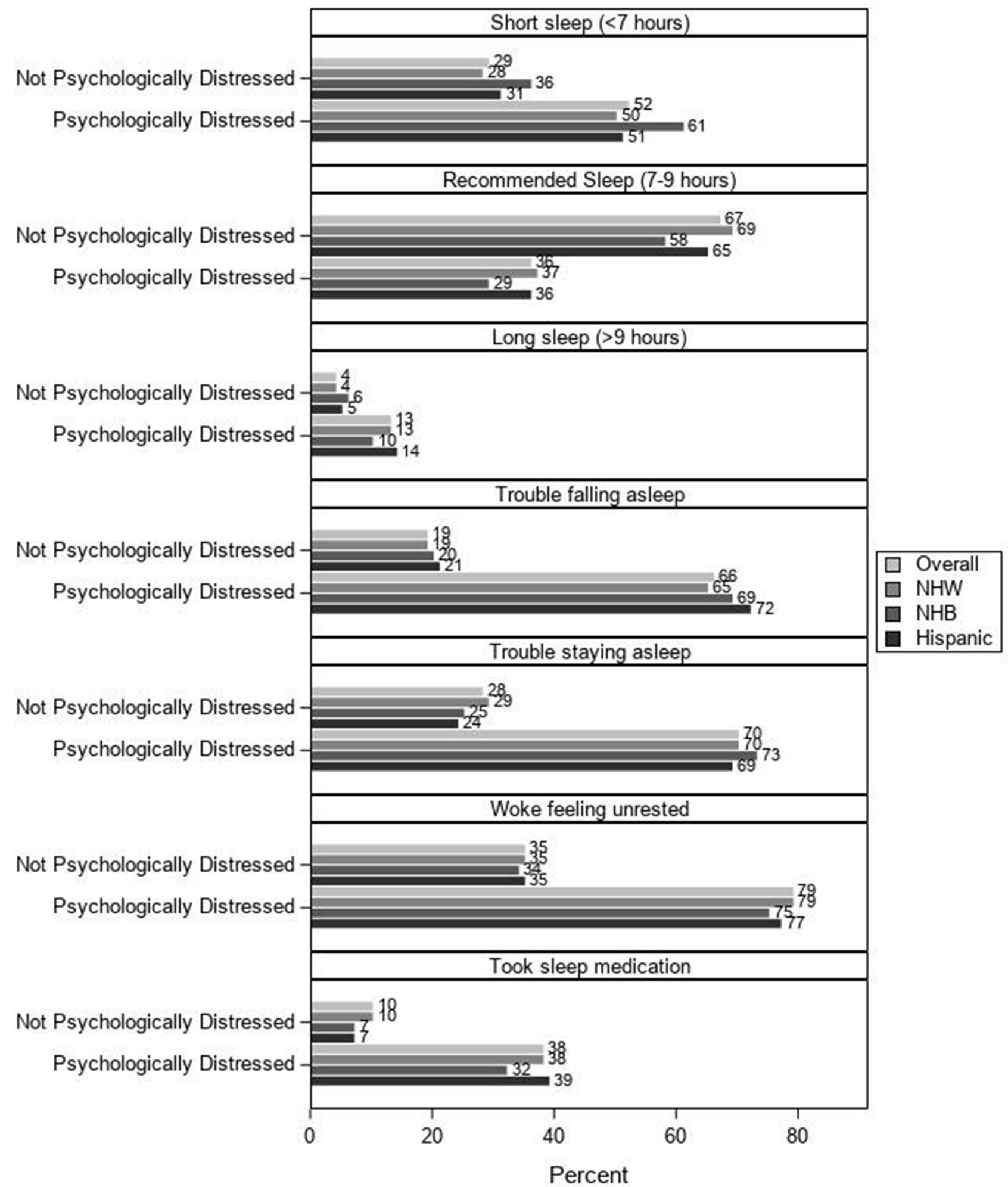

Figure I Prevalence of Sleep Duration and Sleep Disturbances Stratified by Serious Psychological Distress Status among the Overall Population, White, Black, Hispanic/ Latinx Participants, N=316,840.

as having SPD, and that the positive association between short sleep and SPD was strongest among Black participants. Positive associations between sleep disturbances and SPD were generally stronger among Black and Hispanic/Latinx adults compared to White adults. 


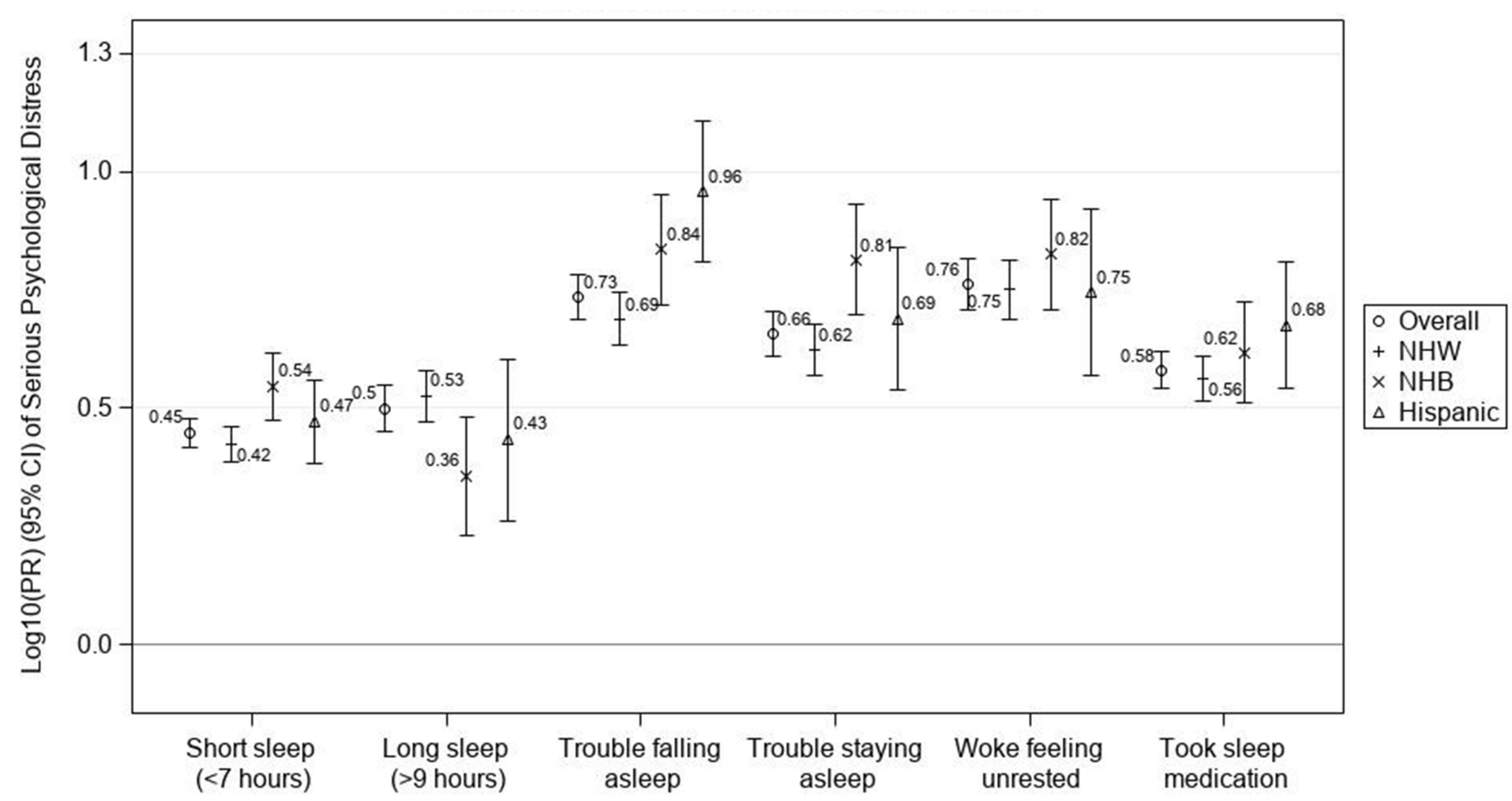

Figure 2 Fully Adjusted Prevalence Ratios for Serious Psychological Distress among Overall, White, Black, and Hispanic/Latinx Participants who Reported Short and Long Sleep Duration along with Sleep Disturbances Compared to their Racial/Ethnic Counterparts with Recommended Sleep, N=316,840.

Notes: Models were adjusted for age, sex/gender, educational attainment, annual household income, occupational class, employment status, region of residence, heavy alcohol consumption and "ideal” cardiovascular health; "Ideal” cardiovascular health includes never smoking/quit $>12$ months prior to interview, BMI I8.5 - <25 kg/m2, high levels of physical activity, and no prior diagnosis of dyslipidemia, hypertension, or diabetes/prediabetes.

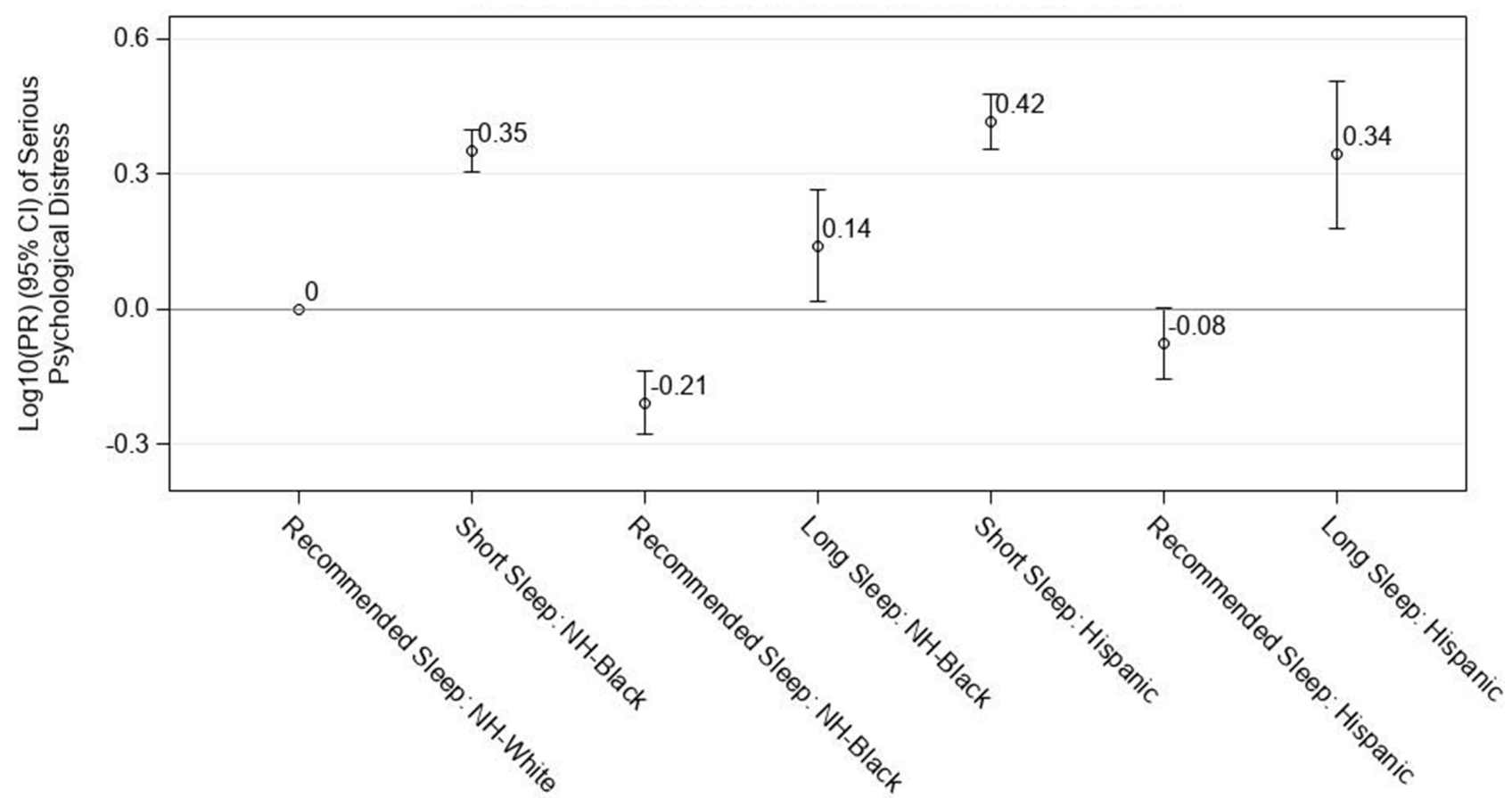

Figure 3 Fully Adjusted Prevalence Ratios for Serious Psychological Distress among NH-Blacks and Hispanics/Latinxs Reporting Short (<7 hours), Recommended (7-9 hours), and Long ( $\geq$ hours) Sleep Duration Compared to NH-Whites with Recommended Sleep, N=3I6,840.

Notes: Models were adjusted for age, sex/gender, educational attainment, annual household income, occupational class, employment status, region of residence, heavy alcohol consumption and "ideal" cardiovascular health; "Ideal" cardiovascular health includes never smoking/quit $>12$ months prior to interview, BMI I8.5 - $<25 \mathrm{~kg} / \mathrm{m}^{2}$, high levels of physical activity, and no prior diagnosis of dyslipidemia, hypertension, or diabetes/prediabetes. 
These findings are consistent with previous research and are relevant because poor sleep health is thought to cause SPD by dysregulating brain areas (eg, the ventrolateral preoptic nucleus and the orexinergic system) that are important for awake to sleep and non-REM to REM transitions as well as mood-regulation. ${ }^{35}$ In a crosssectional study conducted in 2015 by Cunningham et al, the Behavioral Risk Factor Surveillance System was used to cross-sectionally examine the association between various definitions of SPD and short and long sleep duration by sex/gender. Acknowledging the likely bidirectional relationship, the authors found that adults with SPD were more likely to report short and long sleep duration. ${ }^{21}$ Although this study used a different data source, considered SPD as the exposure and short or long sleep duration as the outcome, and did not include sleep disturbances, the significance and magnitude of the associations between poor sleep duration and SPD were comparable to our study. Also similar to our study, a prior study using NHIS data from 2009 observed positive associations between poor sleep and SPD in that Seixas et al found that individuals with SPD had 55\% greater odds of reporting unhealthy sleep. ${ }^{19}$ Previous literature has also identified racial/ethnic disparities in sleep duration and disturbances, which were found in this study. In a meta-analysis of 14 non-overlapping studies, disparities among Black and White US adults were observed for sleep duration. Black adults had lower amounts of time in bed spent sleeping and percentages of time spent in deeper stages of sleep. ${ }^{36}$ Additionally, this meta-analysis found that sleep continuity and duration were moderated by biopsychosocial factors like mental illness. Consistent with this metaanalysis, our results suggested that while the associations between poor sleep health and SPD were significant for all race/ethnicities, the magnitude of the associations were generally stronger among racial/ethnic minorities. Furthermore, the strong association found between long sleep duration and SPD for Whites in our study is inconsistent with the literature that generally found associations between long sleep duration and SPD are strongest among racial/ethnic minorities, which warrants further investigation. $^{20}$

After stratifying by self-rated health, we found that the magnitude of the associations between poor sleep health and SPD were stronger among participants who reported excellent/very good/good health compared to those who reported fair/poor health. This finding underscores the likely connection between SPD and sleep health. Poor sleep health has been consistently associated with poor overall health. ${ }^{37,38}$ The absence of individuals with poor health among participants with excellent/very good/good health likely resulted in a less obscured association between sleep and SPD. This finding further supports the importance of sleep as a potential contributor to SPD.

Differential exposure to various threats to both sleep health and psychosocial stressors that can cause distress by racial/ethnic group likely contribute to the observed disparities in the sleep-SPD relationship. ${ }^{39-41}$ For instance, racial/ethnic minorities are more likely than Whites to be exposed to unique discriminatory practices and policies through institutional, personally mediated, and internalized racism that are embedded in US culture. ${ }^{42-45}$ Discrimination and racism have been linked to both poor sleep and SPD. ${ }^{13,46,47}$ Racial/ethnic disparities in sleep health may also be explained, in part, by the various types of structural factors such as work or school hours that differ among race/ethnicities. ${ }^{48,49}$ For instance, racial/ ethnic minorities have been shown to have disproportionate exposure to financial strain, unemployment, job strain, neighborhood disorder, and substandard housing. ${ }^{50-53}$ Research has shown that exposure to, for instance, poverty, violence, and a lack of social cohesion and integration into society are powerful social determinants of mental illness. ${ }^{4}$ These factors may contribute to disadvantaged communities including racial/ethnic minorities having a higher prevalence of poor sleep health and SPD.

Interestingly, the prevalence of SPD among Blacks and Hispanics/Latinxs with recommended sleep duration was lower compared to the prevalence of SPD among Whites with recommended sleep duration, suggesting that sleep duration may partially explain racial/ethnic differences in SPD. Research is warranted to investigate, for instance, if structural interventions aimed to increase the attainment of recommended sleep among racial/ethnic minorities could reduce the burden of SPD among racial/ethnic minorities. ${ }^{54,55}$

With regard to women, the percentages of women among participants with SPD were larger than the percentage of women found among participants without SPD in our study, which is consistent with prior literature regarding mental health outcomes such as depression. ${ }^{56}$ Women have been found to be more likely than men to seek help for and disclose mental health problems to their healthcare providers while men are more likely to seek specialist mental health care. ${ }^{57}$ 
The limitations of this study include its cross-sectional design, which precludes causal inference as temporality between sleep and SPD cannot be established. The relationship between poor sleep health and SPD is bidirectional (poor sleep health can be treated as an outcome of SPD). Consequently, either getting poor sleep or having SPD might contribute to the poor sleep-SPD relationship. ${ }^{58}$ SPD does not constitute a clinical diagnosis; however, studies have validated its utility among populations with clinically significant anxiety or depression. ${ }^{27}$ All data were self-reported. Generally, self-reported measures of sleep duration overestimate sleep duration data derived from objective measures (eg, actigraphy and PSG). Concordance studies have revealed that self-reported measures of sleep duration and sleep disturbances are moderately to poorly comparable to objective measures such as actigraphy. ${ }^{59-63}$ Of note, it has been shown that such measurement error is nondifferential across racial/ethnic groups depending on the sleep item. ${ }^{64,65}$ Because mental health is stigmatized in the US and globally, it is possible that selfreported measures of SPD could underestimate the true prevalence of SPD, especially among racial/ethnic minority groups. ${ }^{66}$ Sex/gender was also assessed in a binary vs nonbinary manner.

Despite these limitations, strengths of this study include its large sample size where the most recently available data were collected over multiple survey years, which decreases potential influence attributable to singleyear collection periods. The sample size also allowed robust stratification by race/ethnicity. Data also allowed for adjustment of relevant confounders. Furthermore, the data are generalizable to the current US population of White, Black, and Hispanic/Latinx adults.

Future studies will likely benefit from objective measurements of sleep health characteristics (eg, actigraphy with daily diaries) and should consider how psychometric properties of measures that could differ by race/ethnicity potentially contribute to racial/ethnic disparities in sleep health among US adults. Factors such as race/ethnicity and sex/gender are all important in determining how mental health, distress, and disorder are related to one another. ${ }^{67}$ Understanding how environmental factors along with individual sociodemographic, behavioral, and clinical factors influence the effects of poor sleep on SPD will help identify populations at a higher risk of developing SPD. Such information can direct public health efforts towards both prevention and reduction of adverse mental health among vulnerable populations.

\section{Conclusion}

In conclusion, our study found that poor sleep health was positively associated with SPD, and the magnitude of the association was generally stronger among racial/ethnic minorities. Future studies should seek to inform actionable public health and social policy initiatives that aim to address the drivers of both poor sleep and SPD as well as eliminate sleep disparities to achieve social, environmental, and health equity.

\section{Acknowledgments}

This research was presented, in part, at Society for Advancement of Chicanos and Native Americans in Science - The National Diversity in STEM Conference in Honolulu, Hawaii on October 31 - November 2, 2019 and at the State of North Carolina Undergraduate Research and Creativity Symposium in Durham, North Carolina on November 23, 2019. We are grateful to Stacy Mantooth and Erin Knight for assistance with the literature search.

\section{Author Contributions}

All authors contributed to data analysis, drafting or revising the article, have agreed on the journal to which the article will be submitted, gave final approval of the version to be published, and agree to be accountable for all aspects of the work. Authors: Samuel J. Goldstein, Symielle A. Gaston, John A. McGrath, Chandra L. Jackson Study concept and design: CL Jackson Acquisition of data: CL Jackson Statistical Analysis: JA McGrath, SJ Goldstein Interpretation of data: SJ Goldstein, SA Gaston, JA McGrath, CL Jackson Drafting of the manuscript: SJ Goldstein Critical revision of the manuscript for important intellectual content: SJ Goldstein, SA Gaston, J McGrath, CL Jackson Administrative, technical, and material support: CL Jackson Obtaining funding and study supervision: CL Jackson Final Approval: Goldstein SJ; Gaston SA; McGrath JA; Jackson CL.

\section{Funding}

This work was funded by the Intramural Program at the NIH, NIEHS (Z1AES103325-01) and the NIEHS Scholars Connect Program.

\section{Disclosure}

The authors have no conflicts of interest to disclose. 


\section{References}

1. Vos T, Barber RM, Bell B, et al. Global, regional, and national incidence, prevalence, and years lived with disability for 301 acute and chronic diseases and injuries in 188 countries, 1990-2013: a systematic analysis for the Global Burden of Disease Study 2013. Lancet (London, England). 2015;386(9995):743-800.

2. Insel TR. Assessing the economic costs of serious mental illness. $\mathrm{Am}$ J Psychiatry. 2008;165(6):663-665. doi:10.1176/appi.ajp.2008.0803 0366

3. Reeves WC, Strine TW, Pratt LA, et al. Mental illness surveillance among adults in the United States. MMWR Suppl. 2011;60(3):1-29.

4. Kalia M. Assessing the economic impact of stress-the modern day hidden epidemic. Metabolism. 2002;51(6Suppl 1):49-53. doi:10.10 53/meta.2002.33193

5. Liu Y, Wheaton AG, Chapman DP, Cunningham TJ, Lu H, Croft JB. Prevalence of healthy sleep duration among adults-United States, 2014. MMWR Morb Mortal Wkly Rep. 2016;65(6):137-141. doi:10.15585/mmwr.mm6506a1

6. Adam K, Tomeny M, Oswald I. Physiological and psychological differences between good and poor sleepers. J Psychiatr Res. 1986;20(4):301-316. doi:10.1016/0022-3956(86)90033-6

7. Irwin M, Clark C, Kennedy B, Christian Gillin J, Ziegler M. Nocturnal catecholamines and immune function in insomniacs, depressed patients, and control subjects. Brain Behav Immun. 2003;17(5):365-372. doi:10.1016/S0889-1591(03)00031-X

8. Rodenbeck A, Cohrs S, Jordan W, Huether G, Ruther E, Hajak G. The sleep-improving effects of doxepin are paralleled by a normalized plasma cortisol secretion in primary insomnia. A placebo-controlled, double-blind, randomized, cross-over study followed by an open treatment over 3 weeks. Psychopharmacology. 2003;170(4):423-428. doi:10.1007/s00213-003-1565-0

9. Rodenbeck A, Huether G, Ruther E, Hajak G. Interactions between evening and nocturnal cortisol secretion and sleep parameters in patients with severe chronic primary insomnia. Neurosci Lett. 2002;324(2):159-163. doi:10.1016/S0304-3940(02)00192-1

10. Chen JH. Couples' sleep and psychological distress: a dyadic perspective. J Gerontol B Psychol Sci Soc Sci. 2017;73(1):30-39. doi:10.1093/geronb/gbx001

11. Lei J, Fu J, Yap AU, Fu KY. Temporomandibular disorders symptoms in Asian adolescents and their association with sleep quality and psychological distress. Cranio. 2016;34(4):242-249. doi:10.1179/ 2151090315Y.0000000021

12. Seun-Fadipe CT, Mosaku KS. Sleep quality and psychological distress among undergraduate students of a Nigerian university. Sleep Health. 2017;3(3):190-194. doi:10.1016/j.sleh.2017.02.004

13. Vaghela P, Sutin AR. Discrimination and sleep quality among older US adults: the mediating role of psychological distress. Sleep Health. 2016;2(2):100-108. doi:10.1016/j.sleh.2016.02.003

14. Almojali AI, Almalki SA, Alothman AS, Masuadi EM, Alaqeel MK. The prevalence and association of stress with sleep quality among medical students. J Epidemiol Glob Health. 2017;7(3):169-174. doi:10.1016/j.jegh.2017.04.005

15. Humphreys J, Lee K. Sleep disturbance in battered women living in transitional housing. Issues Ment Health Nurs. 2005;26(7):771-780. doi:10.1080/01612840591008401

16. Jiang S, Yan Z, Jing P, Li C, Zheng T, He J. Relationships between sleep problems and psychiatric comorbidities among China's Wenchuan earthquake survivors remaining in temporary housing camps. Front Psychol. 2016;7:1552. doi:10.3389/fpsyg. 2016.01552

17. Kim TK, Lee HC, Lee SG, Han KT, Park EC. The combined effect of sleep duration and quality on mental health among Republic of Korea armed forces. Mil Med. 2016;181(11):e1581-e1589. doi:10.7205/ MILMED-D-15-00538
18. Ohayon M, Wickwire EM, Hirshkowitz M, et al. National Sleep Foundation's sleep quality recommendations: first report. Sleep Health. 2017;3(1):6-19. doi:10.1016/j.sleh.2016.11.006

19. Seixas AA, Nunes JV, Airhihenbuwa CO, et al. Linking emotional distress to unhealthy sleep duration: analysis of the 2009 National Health Interview Survey. Neuropsychiatr Dis Treat. 2015;11:24 25-2430. doi:10.2147/NDT.S77909

20. Seixas AA, Auguste E, Butler M, et al. Differences in short and long sleep durations between blacks and whites attributed to emotional distress: analysis of the National Health Interview Survey in the United States. Sleep Health. 2017;3(1):28-34. doi:10.1016/j. sleh.2016.11.003

21. Cunningham TJ, Wheaton AG, Giles WH. The association between psychological distress and self-reported sleep duration in a population-based sample of women and men. Sleep Disord. 2015;2015:172064. doi:10.1155/2015/172064

22. Centers for Disease Control and Prevention. National health interview survey. September 30, 2019. Available from: https://www.cdc. gov/nchs/nhis/index.htm?CDC_AA_refVal=https $\% 3 \mathrm{~A} \% 2 \mathrm{~F} \% 2 \mathrm{Fwww}$. cdc.gov\%2Fnchs\%2Fnhis.htm. Accessed October 31, 2020.

23. Hale L, Rivero-Fuentes E. Negative acculturation in sleep duration among Mexican immigrants and Mexican Americans. J Immigr Minor Health. 2011;13(2):402-407. doi:10.1007/s10903-0099284-1

24. Jackson CL, Hu FB, Redline S, Williams DR, Mattei J, Kawachi I. Racial/ethnic disparities in short sleep duration by occupation: the contribution of immigrant status. Soc Sci Med. 2014;118:71-79. doi:10.1016/j.socscimed.2014.07.059

25. Alvarez K, Fillbrunn M, Green JG, et al. Race/ethnicity, nativity, and lifetime risk of mental disorders in US adults. Soc Psychiatry Psychiatr Epidemiol. 2019;54(5):553-565. doi:10.1007/s00127-018-1644-5

26. Hirshkowitz M, Whiton K, Albert SM, et al. National Sleep Foundation's sleep time duration recommendations: methodology and results summary. Sleep Health. 2015;1(1):40-43. doi:10.1016/j. sleh.2014.12.010

27. Kessler RC, Barker PR, Colpe LJ, et al. Screening for serious mental illness in the general population. Arch Gen Psychiatry. 2003;60 (2):184-189. doi:10.1001/archpsyc.60.2.184

28. Agriculture USDoHaHSaUSDo. 2015-2020 dietary guidelines for Americans. December 2015.

29. Centers for Disease Control and Prevention. Defining adult overweight and obesity. 2017. Available from: https://www.cdc.gov/obe sity/adult/defining.html. Accessed October 8, 2019.

30. Lloyd-Jones DM, Hong Y, Labarthe D, et al. Defining and setting national goals for cardiovascular health promotion and disease reduction: the American Heart Association's strategic Impact Goal through 2020 and beyond. Circulation. 2010;121(4):586-613. doi:10.1161/ CIRCULATIONAHA.109.192703

31. Blewett LA, Rivera DJA, King ML, Williams KC. IPUMS health surveys: national health interview survey Version 6.4. 2020. Available from: https://www.nhis.ipums.org.

32. Barros AJ, Hirakata VN. Alternatives for logistic regression in cross-sectional studies: an empirical comparison of models that directly estimate the prevalence ratio. BMC Med Res Methodol. 2003;3:21

33. Johnson DA, Thorpe RJ, McGrath JA, Jackson WB, Jackson CL. Black-white differences in housing type and sleep duration as well as sleep difficulties in the United States. Int $J$ Environ Res Public Health. 2018;15(4):564. doi:10.3390/ijerph15040564

34. Paul C, Ayis S, Ebrahim S. Psychological distress, loneliness and disability in old age. Psychol Health Med. 2006;11(2):221-232. doi:10.1080/13548500500262945

35. Riemann D, Krone LB, Wulff K, Nissen C. Sleep, insomnia, and depression. Neuropsychopharmacology. 2020;45(1):74-89. doi:10.10 38/s41386-019-0411-y 
36. Ruiter ME, Decoster J, Jacobs L, Lichstein KL. Normal sleep in African-Americans and Caucasian-Americans: a meta-analysis. Sleep Med. 2011;12(3):209-214. doi:10.1016/j.sleep.2010.12.010

37. Fernandez-Mendoza J, Vgontzas AN. Insomnia and its impact on physical and mental health. Curr Psychiatry Rep. 2013;15(12):418. doi:10.1007/s11920-013-0418-8

38. Grandner MA, Alfonso-Miller P, Fernandez-Mendoza J, Shetty S, Shenoy S, Combs D. Sleep: important considerations for the prevention of cardiovascular disease. Curr Opin Cardiol. 2016;31 (5):551-565. doi:10.1097/HCO.0000000000000324

39. Hicken MT, Lee H, Ailshire J, Burgard SA, Williams DR. "Every shut eye, ain't sleep": the role of racism-related vigilance in racial/ ethnic disparities in sleep difficulty. Race Soc Probl. 2013;5 (2):100-112. doi:10.1007/s12552-013-9095-9

40. Petrov ME, Lichstein KL. Differences in sleep between black and white adults: an update and future directions. Sleep Med. 2016;18:74-81. doi:10.1016/j.sleep.2015.01.011

41. Billings ME, Hale L, Johnson DA. Physical and social environment relationship with sleep health and disorders. Chest. 2020;157:1304-1312.

42. Jackson CL, Redline S, Emmons KM. Sleep as a potential fundamental contributor to disparities in cardiovascular health. Annu Rev Public Health. 2015;36:417-440. doi:10.1146/annurev-publhealth-031914122838

43. Jones CP. Levels of racism: a theoretic framework and a gardener's tale. Am J Public Health. 2000;90(8):1212-1215.

44. Williams DR. Stress and the mental health of populations of color: advancing our understanding of race-related stressors. $J$ Health Soc Behav. 2018;59(4):466-485.

45. Pearce J, Rafiq S, Simpson J, Varese F. Perceived discrimination and psychosis: a systematic review of the literature. Soc Psychiatry Psychiatr Epidemiol. 2019;1-22.

46. Slopen N, Lewis TT, Williams DR. Discrimination and sleep: a systematic review. Sleep Med. 2016;18:88-95. doi:10.1016/j.sleep.2015.01.012

47. Krieger N, Kosheleva A, Waterman PD, Chen JT, Koenen K. Racial discrimination, psychological distress, and self-rated health among US-born and foreign-born Black Americans. Am J Public Health 2011;101(9):1704-1713.

48. Whitehead M. The concepts and principles of equity and health. Int J Health Serv. 1992;22(3):429-445. doi:10.2190/986L-LHQ6-2VTEYRRN

49. Jackson CL, Lee S, Crain T, Buxton O. Bidirectional relationships between work and sleep. In: Kawachi I, Redline S, Duncan D, editors. The Social Epidemiology of Sleep. 1st. Oxford University Press; 2019.

50. Assari S. Race, depression, and financial distress in a nationally representative sample of American adults. Brain Sci. 2019;9(2):29. doi:10.3390/brainsci9020029

51. Wagmiller RL. Race and the spatial segregation of jobless men in urban America. Demography. 2007;44(3):539-562. doi:10.1353/ dem.2007.0032

52. Jacobs DE. Environmental health disparities in housing. Am J Public Health. 2011;101(Supp11):S115-122. doi:10.2105/AJPH.2010.300058

53. Alegría M, Molina KM, Chen CN. Neighborhood characteristics and differential risk for depressive and anxiety disorders across racial/ ethnic groups in the United States. Depress Anxiety. 2014;31 (1):27-37. doi:10.1002/da.22197
54. Bratter JL, Eschbach K. Race/ethnic differences in nonspecific psychological distress: evidence from the National Health Interview Survey. Soc Sci Q. 2005;86(3):620-644. doi:10.1111/j.0038-4941.20 05.00321.x

55. Riolo SA, Nguyen TA, Greden JF, King CA. Prevalence of depression by race/ethnicity: findings from the National Health and Nutrition Examination Survey III. Am J Public Health. 2005;95 (6):998-1000. doi:10.2105/AJPH.2004.047225

56. Salk RH, Hyde JS, Abramson LY. Gender differences in depression in representative national samples: meta-analyses of diagnoses and symptoms. Psychol Bull. 2017;143(8):783-822. doi:10.1037/bul0000102

57. Allen LM, Nelson CJ, Rouhbakhsh P, et al. Gender differences in factor structure of the self-administered alcoholism screening test. J Clin Psychol. 1998;54(4):439-445. doi:10.1002/(SICI)1097-4679(199806)54:4<439::AID-JCLP6>3.0.CO;2-I

58. Garde AH, Albertsen K, Persson R, Hansen AM, Rugulies R. Bidirectional associations between psychological arousal, cortisol, and sleep. Behav Sleep Med. 2011;10(1):28-40. doi:10.1080/15402002. 2012.636272

59. Cespedes EM, Hu FB, Redline S, et al. Comparison of self-reported sleep duration with actigraphy: results from the Hispanic Community Health Study/Study of Latinos Sueno Ancillary Study. Am J Epidemiol. 2016;183(6):561-573. doi:10.1093/aje/kwv251

60. Girschik J, Fritschi L, Heyworth J, Waters F. Validation of self-reported sleep against actigraphy. $J$ Epidemiol. 2012;22 (5):462-468. doi:10.2188/jea.JE20120012

61. Lauderdale DS, Knutson KL, Yan LL, Liu K, Rathouz PJ. Selfreported and measured sleep duration: how similar are they? Epidemiology (Cambridge, Mass). 2008;19(6):838-845. doi:10.10 97/EDE.0b013e318187a7b0

62. van Kooten JA, Terwee CB, Kaspers GJ, van Litsenburg RR. Content validity of the patient-reported outcomes measurement information system sleep disturbance and sleep related impairment item banks in adolescents. Health Qual Life Outcomes. 2016;14:92. doi:10.1186/ s12955-016-0496-5

63. Hanish AE, Lin-Dyken DC, Han JC. PROMIS sleep disturbance and sleep-related impairment in adolescents: examining psychometrics using self-report and actigraphy. Nurs Res. 2017;66(3):246-251. doi:10.1097/NNR.0000000000000217

64. Jackson CL, Patel SR, Jackson WB, Lutsey PL, Redline S. Agreement between self-reported and objectively measured sleep duration among white, black, hispanic, and Chinese adults in the United States: multi-ethnic study of atherosclerosis. Sleep. 2018;41 (6). doi:10.1093/sleep/zsy057

65. Jackson CL, Ward JB, Johnson DA, Sims M, Wilson J, Redline S. Concordance between self-reported and actigraphy-assessed sleep duration among African-American adults: findings from the Jackson Heart Sleep Study. Sleep. 2020;43(3). doi:10.1093/sleep/zsz246

66. Thompson VLS, Bazile A, Akbar M. African Americans' perceptions of psychotherapy and psychotherapists. Prof Psychol Res Pr. 2004;35 (1):19-26. doi:10.1037/0735-7028.35.1.19

67. Payton AR. Mental health, mental illness, and psychological distress: same continuum or distinct phenomena? J Health Soc Behav. 2009;50 (2):213-227. 


\section{Publish your work in this journal}

Nature and Science of Sleep is an international, peer-reviewed, open access journal covering all aspects of sleep science and sleep medicine, including the neurophysiology and functions of sleep, the genetics of sleep, sleep and society, biological rhythms, dreaming, sleep disorders and therapy, and strategies to optimize healthy sleep.
The manuscript management system is completely online and includes a very quick and fair peer-review system, which is all easy to use. Visit http://www.dovepress.com/testimonials.php to read rea quotes from published authors. 